East African Medical Journal Vol. 85 No. 11 November 2008

HIV-ASSOCIATED PULMONARY HYPERTENSION: CASE REPORT

J. Shavadia, MBChB, Resident, S. Das, MRCP, Registrar and G. Yonga, FESC, FACC, Physician and Cardiologist, Aga

Khan University Hospital Nairobi, P. O. Box 40374-00100, Nairobi, Kenya

Request for reprints to: Dr. J. Shavadia, The Aga Khan University Hospital, P. O. Box 40374-00100, Nairobi, Kenya

\title{
HIV-ASSOCIATED PULMONARY HYPERTENSION: CASE REPORT
}

\author{
J. SHAVADIA, S. DAS and G. YONGA
}

\section{SUMMARY}

\begin{abstract}
With the advent of highly active antiretroviral therapy, there has been a significant change in the epidemiology of pulmonary disease in HIV/AIDS. The relative prevalence of non-infectious manifestations is likely to rise. HIV associated pulmonary hypertension (HIV-PH), albeit low prevalence, is associated with significant morbidity and mortality. Presently, despite having scanty evidence on the management modalities of HIV-PH, evidence extrapolated from idiopathic pulmonary hypertension is being utilised to effectively manage some of these patients. Efforts should therefore be made to screen, diagnose and treat these patients. A case of a thirty year old female with HIV disease and severe pulmonary hypertension is presented.
\end{abstract}

\section{INTRODUCTION}

Since the description of HIV associated pulmonary hypertension (HIV-PH) by Kim and Factor in 1987, the prevalence of HIV-PH has been reported to be between $0.06-2 \%$ (1). This is farless common compared to the infectious complications associated with HIV disease. However, with widespread antiretroviral therapy (ART) rollout programmes, more people are now living with HIV hence the relative prevalence of non-infectious complications related to HIV disease are likely to increase.

HIV-PH has been documented to occur at all stages of the disease and its manifestations range from asymptomatic right ventricular dysfunction to overt right heart failure. There have been no large scale studies on the effects of ART on HIV-PH. Most treatment modalities currently in place are an extension of evidence from trials used to manage idiopathic pulmonary hypertension.

We present a case of HIV-PH to highlight the importance of the non infectious pulmonary complications associated with HIV disease.

\section{CASE REPORT}

A thirty year old female presented with an eight month history of progressively increasing shortness of breath at The Aga Khan University Hospital, Nairobi on the $29^{\text {th }}$ January 2008. She also reported associated lower limb swelling, facial puffiness and difficulty in lying on a flat bed. In the last month prior to her admission, the shortness occurred even during bathing and dressing. She also had a nocturnal non productive cough. She was para $3+0$ and all her pregnancies were uneventful. She denied any history of a wheeze, haemoptysis, rash, oral or genital sores. She had no history of febrile illness, night sweats or weight loss. She had been started on frusemide and candensartan for presumed heart failure at the nearest health facility and reported temporary relief of symptoms, until one month prior to presentation.

Examination revealed an afebrile ill looking young lady whose vital signs were as: $\mathrm{BP} 110 / 62 \mathrm{mmHg}$ supine, P132 bpm regular, of low volume and normal character, RR 20 breaths / min. General examination revealed oral candidiasis and bipedal lower limb pitting oedema. No joint swelling or deformity and no skin rashes were found.

Pertinent cardiovascular findings included an elevated jugular venous pulsation with a prominent $\mathrm{v}$ wave. Her apex beat was undisplaced and her cardiac impulse felt normal. She had a left lower parasternal lift and a palpable P2. Cardiac auscultation confirmed a loud $\mathrm{P} 2$ and a pansystolic murmur in the left lower sternal border which was accentuated on inspiration. The rest of her systemic exam was non contributory. A clinical diagnosis of right heart failure with pulmonary hypertension and immunosuppression was made.

A resting 12-lead ECG demonstrated an incomplete right bundle branch block and evidence of right atrial overload. Echocardiogram revealed a moderately enlarged right ventricle and atrium with paradoxical septal motion. She had moderate- severe tricuspid regurgitation with an estimated pulmonary 
arterial pressure of $80 \mathrm{mmHg}$. Her left ventricular ejection fraction was 50\% with normal mitral and tricuspid valve anatomy. No atrial septal defect or any other cardiac shunts were found.

Full blood count and ESR, renal and liver functions were normal, and her peripheral blood film did not reveal any evidence of an underlying haemolytic disorder. After counselling and testing, an ELISA for HIV was positive, CD4 count was 312cells / $\mathrm{mm}^{3}$ and a plasma viral load 75,000 copies / $\mathrm{mm}^{3}$. She was hepatitis B surface antigen negative. A CT pulmonary angiogram ruled out pulmonary thromboembolic disease.

She was commenced on diuretics, antifungals, anticoagulation and later sildenafil introduced. She experienced significant symptomatic improvement. On the fifth hospitalisation day the patient was able to ambulate freely in the ward, and perform her personal activities without significant limitation. She was discharged home on $5^{\text {th }}$ February 2008, eight days after hospitalisation on frusemide, warfarin, tenofovir/emtricitabine and efavirenz.

On out-patient follow up three months later, the patient reported to be able to climb two flights of stairs and perform her activities of daily living comfortably. She was to have an echocardiogram repeated in 12 months from her initial presentation for estimation of her pulmonary arterial pressures as a follow up of the efficacy of treatment. The frequency of echocardiographic follow-up was based on the patient living away from Nairobi, and concomitant financial strain on performing six monthly echocardiographic assessments. However, improvement in her dyspnoea severity, as assessed by her New York Heart Association criteria, about three months into therapy is a strong clinical indicator of therapeutic efficacy and this parameter in addition to yearly echocardiography will be utilised for her follow up.

\section{DISCUSSION}

Pulmonary hypertension $(\mathrm{PH})$ is a relentlessly progressive disease leading to right heart failure and death. With a worldwide prevalence of HIV disease estimated at 40 million (2), and that of HIV-PHat0.06$2.0 \%$ (1), HIV disease maybeamong the most common cause of pulmonary hypertension worldwide.

Reports from other parts of Africa, however, suggest a much higher prevalence of HIV-PH at about $5 \%$ (3). This goes to reflect the potential magnitude of HIV -PH in sub Saharan Africa, which houses over two-thirds of the global HIV burden (4). Despite these alarming figures, a pubmed search did not reveal any citations from Kenya on the prevalence of this complication associated with HIV disease.

A diagnosis of HIV-PH was made in this patient after exclusion of the common secondary causes of $\mathrm{PH}$, as connective tissue disease, chronic thromboembolic disease and haemolytic anaemias. Due to the age bracket the index patient presented in, connective tissue disease fell high on the list. However, her clinical history, physical examination and supportive laboratory and radiological evidence were not in keeping for a connective tissue disease, chronic thromboembolism or haemolytic anaemias. Since the potential causes for $\mathrm{PH}$ are numerous and investigations to rule out each would be superfluous, it is imperative to obtain a good clinical history and perform a thorough clinical evaluation to narrow the differential causative agents as performed in this patient.

Pulmonary arterial hypertension, of which $\mathrm{HIV}-\mathrm{PH}$ is a sub entity, defines an increase in mean pulmonary arterial pressure related to an arteriopathy of the pulmonary vasculature. Patients with HIV -PH have plexogenic lesions similar to diseases associated with pulmonary arterial hypertension.

Majority of the patients clinically present with dyspnoea on exertion (85\%), pedal oedema (20-30\%) and a non-productive cough (19\%). The interval duration between symptom development and diagnosis is reported at six months (5). This data is in line with the clinical symptoms and duration between symptom onset and diagnosis in the index patient. There is also no correlation between the CD4 count or plasma viral load and the development of HIV-PH $(6,7)$.

In terms of pathogenetic mechanisms, there is noevidence incriminating human immunodeficiency virus (HIV) to be directly infecting pulmonary vascular endothelial cells $(8,9)$. However, viral antigens such as GP120 and Nef, are thought to stimulate growth and proliferation $(8,10,11)$. The other plausible mechanism includes induction by HIV infection of a chronic inflammatory state with release of growth promoting cytokines which stimulate smooth muscle hypertrophy and increase pulmonary vascular resistance $(12,13)$.

Treatment of patients with HIV-PH is based on symptomatology, functional class and the severity of $\mathrm{PH}$. Although vasodilator agents increase survival in patients with idiopathic pulmonary hypertension, the impact of these therapies on HIV-PH is unknown $(14,15)$.

Nunes et al (16) reported better survival in patients on epoprostenol infusion. In another smaller study, Petitpretz et al (6) also reported significant improvement in the haemodynamics in patients on epoprostenol. Epoprostenol has well described adverse effects, as well as its difficulty in administration. We therefore chose sildenafil, a phosphodiesterase-5-inhibitor, which has shown some short term efficacy in case reports of patients with HIV-PH (17-19). A potential problem with sildenafil is that its levels may significantly increase by concurrent use of protease inhibitors, hence the choice of efavirenz in our patient. 
The other potential choice to reduce pulmonary arterial pressurewouldincludeendothelinantagonists, as bosentan. Like all the other agents, scanty data exists in HIV-PH (20-22). In view of this, its unavailability in our region, and the exorbitant costs of the drug, our patient was not started on it.

\section{Figure 1}

Apical 4 chamber view demostrating an enlarged right atrium and enlarged right atrium and ventricle

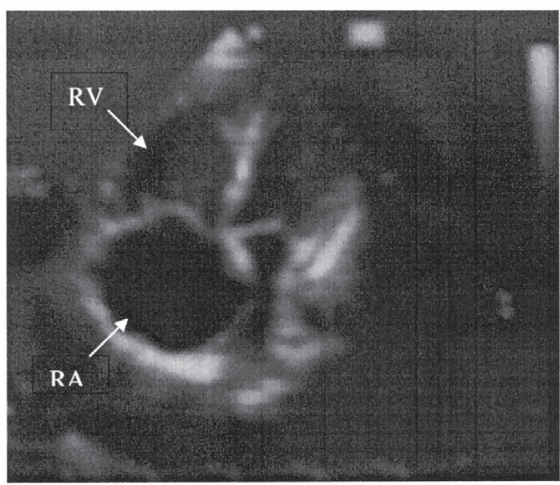

The role of antiretroviraI therapy in patients with HIV-PH is not clear since large scale trials are lacking. Conflicting evidence exists in literature on the role of ART in HIV-PH. Zuber et al (1) in their retrospective study showed a decreased incidence of PH following the introduction of HAART; in addition, they also demonstrated reduced mortality associated with PH. However, other studies $(7,14)$ failed to demonstrate an effect of ART on HIV-PH. Nunes et al (7) observed that survival rates were worse in patients receiving HAART alone compared to HAART and epoprostenol. Therefore until well conducted multicenter prospective studies are conducted the survival benefit of HAART in patients with HIV -PH cannot be definitively concluded.

\section{Figure 2}

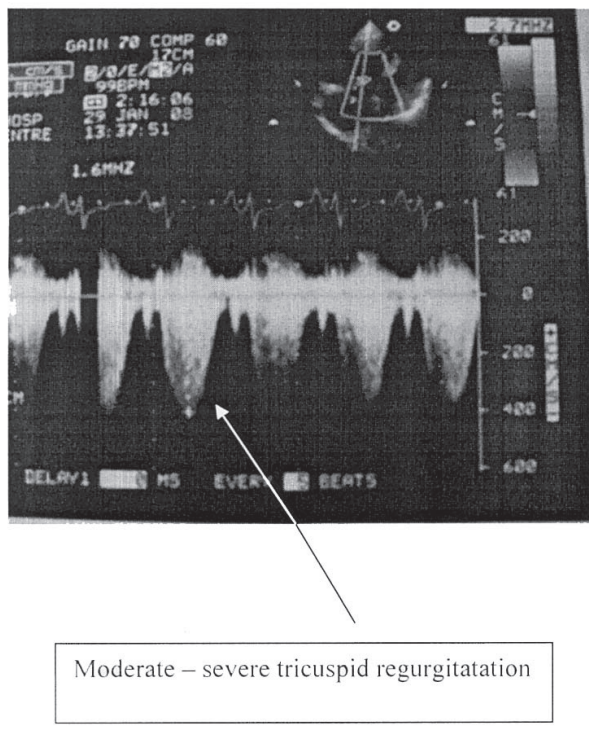

This case highlights the presence of HIV-PH whose mortality is two fold higher in HIV patients without pulmonary hypertension (23). It also brings to the fore the potential problems our HIV patients may now present with, since a large proportion of patients on HAART are now living much longer with HIV disease. It also calls for larger studies in our region to build up regional evidence in order to better the management of such patients.

\section{REFERENCES}

1. Zuber, J. P., Calmy, A., Evison, J. M. et al. Pulmonary arterial hypertension related to HIV infection: improved hemodynamics and survival associated with antiretroviral therapy. Clin. Infect. Dis. 2004; 38: 1178-1185.

2. Ministry of Health Kenya, 2005; National AlDS Control Council Kenya, 2007.

3. Niakara, A., Orabo, Y. J., Kambire, Y. et al. Cardiovascular diseases and HIV infection: study of 79 cases at the National Hospital of Ouagadougou (Burkina Faso). Bull. Soc. Pathol. Exot. 2002; 95: 23-26.

4. UNAIDS 2007

5. O'Alonzo, G. E., et al. Survival in patients with primary pulmonary hypertension. Results from a national prospective registry. Ann. Intern. Med. 1991; 115: 343-349.

6. Petitpretz, P., et al. Pulmonary hypertension in patients with human immunodeficiency virus infection. Comparison with primary pulmonary hypertension. Circulation. 1994; 89: 2722-2727.

7. Nunes, H., Humbert, M., Sitbon, O., et al. Prognostic factors for survival in human immunodeficiency virus-associated pulmonary arterial hypertension. Am. J. Respir. Crit. Care. Med. 2003; 167: 1433-1439.

8. Kanmogne, G. O., Primeaux, C. and Grammas, P. Induction of apoptosis and endothelin-I secretion in primary human lung endothelial cells by HIV-lgp120 proteins. Biochem. Biophys. Res. Commun. 2005; 333: 1107-1115.

9. Humbert, M., Monti, G., Fartoukh, M., et al. Plateletderived growth factor expression in primary pulmonary hypertension: comparison of HIV seropositive and HIV seronegative patients. Eur. Respir. J. 1998; 11: 554-559.

10. Marecki, J. C., Cool, C. D., Parr, J. E., et al. HIV-1 Nefis associated with complex pulmonary vascular lesions in SHIV-Nef-infected macaques. Am. J. Respir. Crit. Care. Med. 2006; 174: 437-445.

11. Marecki, J. C., Cool, C. D., Beckey, V. E., et al. HIV-1 Nefprotein is present a the site of plexiform lesions in patients with HIV -related pulmonary hypertension, and induces a program of altered endothelial cell growth and survival in vitro. Proc. Am. Thorac. Soc. 2006; 3: A476.

12. Fauci, A. S., Pantaleo, G., Stanley, S. and Weissman, D. Immunopathogenic mechanisms of HIV infection. Ann. Intern. Med. 1996; 124: 654-663.

13. Morse, J. H., Barst, R. J., ltescu, S., et al. Primary pulmonary hypertension in HIV infection: an outcome determined by particular HLA class II alleles. Am. J. Respir. Crit. Care. Med. 1996; 153: 1299-1301. 
14. Pellicelli, A. M., et al. Pathogenesis of HIV-related pulmonary hypertension. Ann. N.Y. Acad. Sci. 2001; 946: 82-94.

15. Tuder, R. M., et al. Exuberant endothelial cell growth and elements of inflammation are present in plexiform lesions of pulmonary hypertension. Am. J. Pathol. 1994; 144: 275-285.

16. Nunes, H., et al. Prognostic factors for survival in human immunodeficiency virus-associated pulmonary arterial hypertension. Am. J. Respir. Crit. Care. Med. 2003; 167: 1433-1439.

17. Prasad,S., Wilkinson, J. and Gatzoulis, M. A. Sildenafil in primary pulmonary hypertension. N. Engl. J. Med. 2000; 343: 1342.

18. Schumacher, Y. O., et al. Sildenafil in HIV-related pulmonary hypertension. AIDS. 2001; 15: 1747-1748.

19. Alp, S., et al. Long-time survival with HIV-related pulmonary arterial hypertension: a case report. AIDS. 2003; 17: 1714-1715
20. Sitbon, O., et al. Bosentan for the treatment of human immunodeficiency virus-associated pulmonary arterial hypertension. Am. J. Respir. Crit. Care. Med. 2004; 170: 1212-1217.

21. Cea-Calvo, L., et al. Treatment of HIV-associated pulmonary hypertension with treprostinil. Rev. Esp. Cardiol. 2003; 56: 421- 425.

22. Channick, R. N., et al. Effects of the dual endothelinreceptor antagonist bosentan in patients with pulmonary hypertension: a randomised placebocontrolled study. Lancet. 2001; 358: 1119 - 1123.

23. Opravil, M., Pechere, M., Speich, R., et al. HIVassociated primary pulmonary hypertension: a case control study: Swiss HIV Cohort Study. Am. J. Respir. Crit. Care. Med. 1997; 155: 990-995. 


\section{GOOD CLINICAL LABORATORY PRACTICE (GCLP) COURSE AT KENYA AIDS VACCINE INITIATIVE}

\section{Description:}

This course is designed to prove comprehensive guidance and practical help for those who are implementing Good Clinical Laboratory Practice in laboratories which undertake the analysis of samples from clinical trials.

The course will address the current regulatory framework for laboratory work in support of clinical trials with reference to the ICH Guideline for Good Clinical Practice, the Clinical EU Trials Directive and related regulations and guidance. The course will also draw on the BARQA guidance document on Good Clinical Laboratory Practice (GCLP).

The course is structured to encourage delegates to:

$\sqrt{ }$ Discuss and develop ideas.

$\sqrt{ }$ Solve specific problems.

$\sqrt{ }$ Examine particular aspects of GCP and GCLP.

\section{Benefits include:}

$\sqrt{ }$ Guidance on the interpretation and application of GCLP within the framework of Good Clinical Practice (GCP).

$\sqrt{ }$ Understand how GCLP fits within a clinical (GCP) programme.

$\sqrt{ }$ Practicalhelp on how to implementGCLP within a clinical research laboratory. $\sqrt{ }$ Anopportunity toupdateyourknowledge of GCLP with the current interpretation of requirements

$\sqrt{ }$ Access to an experienced panel of speakers.

$\sqrt{ }$ An opportunity to improve your understanding of the GCLP requirements as they are applied in different situations.

\section{Who should attend?}

Laboratory managers, analysts, investigators, trial co-ordinators, monitors and auditors working in:

$\sqrt{ }$ Pharmaceutical company laboratories

$\sqrt{ }$ Central laboratories

$\sqrt{ }$ Contract Research Organisations

$\sqrt{ }$ Hospital Laboratories

$\sqrt{ }$ Clinics

$\sqrt{ }$ Investigator sites

\section{Contacts:}

Kenya Aids Vaccine Initiative,

P. O. Box 19676-00202, Nairobi

Tel: $2725405 / 2717694 / 0722207417$

Fax: 2714613

Email: fbashir@kaviuon.org

Website: www.kaviuon.org

Duration of the course: 4 DAYS

Minimum No. of participants: 10 